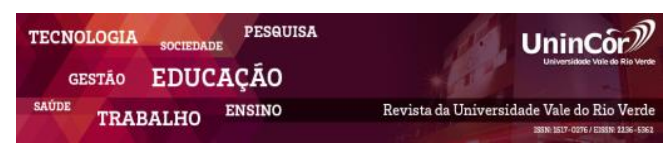

Revista da Universidade Vale do Rio Verde ISSN: 1517-0276 / EISSN: 2236-5362 v. 17 |n. 1 | Ano 2019

Jocias Maier Zanatta

Universidade Federal de Santa Maria - UFSM josk85@hotmail.com

Ismael Alan Halberstadt

Universidade Federal de Santa Maria - UFSM ismaah@hotmail.com

Diéssica de Oliveira Dias Universidade Federal de Santa Maria - UFSM diessica_dias@hotmail.com

Marcelo Trevisan Universidade Federal de Santa Maria - UFSM marcelotrev@gmail.com

\section{ANÁLISE CRÍTICA DA RDC-222/2018 À LUZ DAS DIMENSÕES DO DESENVOLVIMENTO SUSTENTÁVEL: AVANÇOS E DESAFIOS}

\section{RESUMO}

Diante de um cenário de incertezas econômicas, políticas, e de crise da saúde pública e do meio ambiente, é crescente a busca por soluções para a saúde e a sustentabilidade. O presente estudo tem o objetivo de realizar uma análise crítica da RDC-222/2018 à luz das dimensões do desenvolvimento sustentável (ambiental, social e econômica) a fim de verificar os avanços e desafios da legislação. Quanto ao caminho metodológico, o estudo se define como de natureza exploratória e quanto aos procedimentos foi adotada a análise documental. Discorreu-se sobre as legislações de resíduos, aspectos de sustentabilidade e desenvolvimento sustentável e concluiu-se que a nova resolução é um avanço importante em busca da regulamentação e das boas práticas no tratamento de resíduos de serviços de saúde em convergência as dimensões do desenvolvimento sustentável, apesar da dificuldade na fiscalização e cumprimento da legislação.

Palavras-chave: Desenvolvimento Sustentável, RDC-306/2004, Programa Nacional de Resíduos Sólidos (PNRS), RDC222/2018.

\section{CRITICAL ANALYSIS OF RDC-222/2018 IN THE LIGHT OF SUSTAINABLE DEVELOPMENT DIMENSIONS: ADVANCES AND CHALLENGES}

\section{ABSTRACT}

Faced with a scenario of economic, political, and public health and environmental uncertainties, the search for solutions to health and sustainability is growing. The present study aims to conduct a critical analysis of RDC-222/2018 in light of the dimensions of sustainable development (environmental, social and economic) in order to verify the advances and challenges of the legislation. As for the methodological path, the study is defined as an exploratory nature and as for the procedures, documentary analysis was adopted. The waste legislation, sustainability and sustainable development aspects were discussed and it was concluded that the new resolution is an important advance in the search for regulation and good practices in the treatment of waste from health services in convergence the dimensions of sustainable development, despite the difficulty in monitoring and complying with the legislation.

Keywords: Sustainable Development, RDC-306/2004, National Solid Waste Program (PNRS), RDC-222/2018 
Recebido em: 30/07/2018 - Aprovado em: 09/04/2019 - Disponibilizado em: 15/07/2019

\section{INTRODUÇÃO}

$\mathrm{Na}$ atualidade, nos depararmos com um cenário de incertezas econômicas e políticas (DAPPER;GRENZEL;ZANATTA, 2018) e, há muito tempo, existe uma crise da saúde pública e do meio ambiente, em que sua convergência amplia o poder destrutivo de cada uma (KARLINER; GUENTHER, 2013). Com tudo, observa-se uma crescente busca por soluções que promovam tanto a saúde pública como a sustentabilidade, e neste aspecto, os Resíduos de Serviços de Saúde (RSS) são tema central para os gestores de saúde, em busca de políticas e práticas sustentáveis, uso consciente de recursos naturais e melhor alocação de recursos monetários (NOGUEIRA;CASTILHO, 2016).

De acordo com a Associação Brasileira de Empresa de Limpeza Pública e Resíduos Especiais (ABRELPE), no ano de 2016 o Brasil gerou 71,3 milhões de toneladas de resíduos sólidos urbanos e 256.238 mil toneladas de resíduos de serviço de saúde (ABRELPE, 2016).

A partir desse expressivo quantitativo de resíduos gerados anualmente se justifica uma reflexão crítica acerca da relação entre as políticas públicas e normas existentes com as dimensões do desenvolvimento sustentável (ambiental, social e econômica), sobre tudo, dos resíduos de serviços de saúde. Por isso, será apresentado neste estudo a Política Nacional de Resíduos Sólidos (PNRS), a Resolução da Diretoria Colegiada (RDC) 306/2004, a Resolução da Diretoria Colegiada (RDC) 222/2018 e a sustentabilidade e desenvolvimento sustentável.
O presente estudo tem o objetivo de realizar uma análise crítica da Resolução da Diretoria Colegiada (RDC) 222/2018 à luz das dimensões do desenvolvimento sustentável (ambiental, social e econômica) a fim de verificar os avanços e desafios da legislação. Para tanto o artigo está estruturado em cinco sessões, inicialmente foi realizada uma breve introdução, na segunda sessão foi abordado a Política Nacional de Resíduos Sólidos (PNRS), RDC 306/2004, RDC 222/2018 e a temática da sustentabilidade e desenvolvimento sustentável, em seguida foi apresentado o caminho metodológico, e na sequência se tem a análise e discussão dos resultados, e por fim, as considerações finais do estudo.

\section{LEGISLAÇÕES SOBRE RESÍDUOS}

Nesta sessão é apresentada a Política Nacional de Resíduos Sólidos (PNRS), a Resolução da Diretoria Colegiada (RDC) 306/2004, a Resolução da Diretoria Colegiada (RDC) 222/2018 e sustentabilidade e desenvolvimento sustentável, com o objetivo de compreender os avanços e desafios da legislação e sua relação com as dimensões do desenvolvimento sustentável.

\subsection{Política nacional de resíduos sólidos (PNRS)}

Criada em 2 de agosto de 2010, a lei que estabelece a Política Nacional de Resíduos Sólidos tem como principais objetivos o estabelecimento de normas e regras para com o descarte, reaproveitamento e gestão de resíduos sólidos gerados tanto por instituições públicas, privadas e 
população em geral. A presente lei integra-se com a Política Nacional de Meio Ambiente e articulase com a Política Nacional de Educação Ambiental, em consonância com a Lei n ${ }^{\circ}$ 9.795, de 27 de abril de 1999, com a Política Federal de Saneamento Básico, regulada pela lei $\mathrm{n}^{\mathrm{o}} 11.445$ de 2007, e com a Lei $\mathrm{n}^{\circ} 11.107$, de 6 de abril de 2005 (BRASIL, 2010).

Aspectos como a responsabilidade compartilhada são amplamente discutidos, dentro deste conceito o ciclo de vida dos produtos é de responsabilidade de toda sua respectiva cadeia de produção, distribuição, consumo e destinação pós consumo, responsabilizando todos aqueles que participam diretamente destas cadeias, inclusiva a esfera pública. Fato é que a lei não é muito específica quanto às punições ou restrições caso as determinações não sejam cumpridas e em alguns nichos específicos ela regulamenta de forma impositiva que as organizações que comercializam determinados produtos são totalmente responsáveis pela logística reversa dos mesmos.

Produtos como agrotóxicos, resíduos e embalagens ou produtos demais produtos cuja embalagem possa contaminar o meio ambiente, pilhas e baterias, pneus, óleos lubrificantes, lâmpadas fluorescentes e produtos eletroeletrônicos e seus componentes possuem, segundo a PNRS 2010 responsabilidade de logística reversa outorgada aos responsáveis pela distribuição e comercialização destes produtos. De tal forma que acaba por exonerar do estado a responsabilidade de logística reversa de determinados produtos.

Outro aspecto relevante é a forma como a terminologia "logística reversa" é tratada sob a ótica do estado. Sendo definida como:
"Instrumento de desenvolvimento econômico e social caracterizado por um conjunto de ações, procedimentos e meios destinados a viabilizar a coleta e a restituição dos resíduos sólidos ao setor empresarial, para reaproveitamento, em seu ciclo ou em outros ciclos produtivos, ou outra destinação final ambientalmente adequada" (Art. $3^{\circ}$, item XII)

Sendo assim, a responsabilidade da logística reversa recai tanto para o estado quanto para com as organizações privadas, com exceção de alguns produtos específicos, citados anteriormente. Observa-se que a PNRS não trata diretamente sobre os Resíduos de Serviços de Saúde pois estes possuem normas técnicas específicas devido à sua natureza e riscos ambientais e de saúde envolvidos.

2.2 Resolução da diretoria colegiada (RDC) 306 de 2004

A RDC 306 dispõe sobre o regulamento técnico para o gerenciamento de resíduos de serviços de saúde, criada em 7 de dezembro de 2004 entrou em vigor após 180 dias de sua publicação no Diário Oficial da União (DOU), valendo à partir de 7 de junho de 2005. São tratados como geradores de RSS:

"todos os serviços relacionados com o atendimento à saúde humana ou animal, inclusive os serviços de assistência domiciliar e de trabalhos de campo; laboratórios analíticos de produtos para saúde; necrotérios, fune- 
rárias e serviços onde se realizem atividades de embalsamamento (tanatopraxia e somatoconservação); serviços de medicina legal; drogarias e farmácias inclusive as de manipulação; estabelecimentos de ensino e pesquisa na área de saúde; centros de controle de zoonoses; distribuidores de produtos farmacêuticos, importadores, distribuidores e produtores de materiais e controles para diagnóstico in vitro; unidades móveis de atendimento à saúde; serviços de acupuntura; serviços de tatuagem, dentre outros similares." (RDC 306, Cap. II)

A RDC dispõe também sobre a responsabilidade que todo o gerador de RSS tem sobre a elaboração de Plano de Gerenciamento de Resíduos de Serviços de Saúde (PGRSS). Este documento deve apontar as práticas e ações relativas aos RSS e de que forma a organização maneja, segrega, armazena, transporta e descarta tais resíduos. Outrossim, deverão ser observadas as demais normas técnicas como segurança do trabalho, Comissão de Controle de Infecção Hospitalar (CCIH), Comissões internas de Biossegurança, serviços de Engenharia de Segurança e Medicina do Trabalho (SESMT), Comissão interna de prevenção de acidentes (CIPA) e ainda, caso tenha resíduos de caráter radioativo observar as resoluções da Comissão Nacional de Energia Nuclear (CNEN).

2.2.1 Classificação e manejo dos principais grupos de RSS, segundo a RDC 306 de 2004

Na sequência é apresentada a classificação e manejo dos principais grupos de RSS con- forme a RDC 306/2004: Grupo A1, Grupo A2, Grupo A3, Grupo A4, Grupo A5, Grupo B, Grupo C, Grupo D e Grupo E.

Grupo A1: são culturas e estoques de microrganismos resíduos de fabricação de produtos biológicos, exceto os hemoderivados, resíduos de laboratório de manipulação genética. O acondicionamento, caso não haja descaracterização dos resíduos, deve ser feito em saco branco leitoso, sendo substituído a cada 24 h ou sempre que atingir 2/3 do nível máximo do recipiente.

Grupo A2: São carcaças, peças anatômicas, vísceras e outros resíduos provenientes de animais submetidos a processos de experimentação com inoculação de microrganismos, como como suas forrações e os cadáveres de animais suspeitos de serem portadores de microrganismos de relevância epidemiológica e com risco de disseminação, que foram submetidos ou não a estudo anátomo-patológico ou confirmação diagnóstica. Após o tratamento pode ser destinado à cremação, ao cemitério de animais ou à disposição final em aterro sanitário. Observando o transporte, por meio de saco branco leitoso, com no máximo 2/3 de sua capacidade preenchida e selado (trocado) no máximo em $24 \mathrm{~h}$.

Grupo A3: corresponde a peças anatômicas do ser humano, produtos de fecundação sem sinais vitais, com peso inferior a 500 gramas ou estatura menor de 25 ou idade gestacional inferior a 20 semanas, que não tenham valor científico ou legal e não tenha havido requisição por pacientes ou familiares.

Grupo A4: contempla kits de linhas arteriais, endovenosas e dialisadores, filtros de ar e 
gases aspirados de área contaminada, membrana filtrante de equipamento médico-hospitalar e de pesquisa, sobras de amostras de laboratório e seus recipientes contendo fezes, urina e secreções provenientes de pacientes que não contenham e nem sejam suspeitos de conter agentes de Classe de Risco 4 (contaminantes individuais e de grupos de indivíduos). Estes resíduos podem ser dispostos sem tratamento prévio, em local devidamente licenciado para disposição final de RSS. Devem ser acondicionados em saco branco leitoso, este deve ser trocado a cada $24 \mathrm{~h}$ ou sempre que atingir $2 / 3$ de sua capacidade máxima.

Grupo A5: são órgãos, tecidos, fluídos orgânicos, materiais perfurocortantes ou escarificantes de demais materiais resultantes da atenção à saúde de indivíduos ou animais, com suspeita ou certeza de contaminação com príons.

Devem ser sempre encaminhados a sistema de incineração, de acordo com o definido na RDC ANVISA no 305/2002. Devem ser acondicionados em saco vermelho, que devem ser substituídos sempre que atingir $2 / 3$ de sua capacidade e ao final de cada procedimento.

Grupo B: são resíduos químicos que não possuem destinação institucionalizada por lei, não se aplica a remédios. Resíduos químicos que não apresentam riscos à saúde e ao meio ambiente, quando não forem submetidos a processo de reutilização, recuperação ou reciclagem devem ser submetidos a tratamento ou disposição final em aterros de resíduos perigosos Classe I. Resíduos gerados pelo atendimento a domicílio devem ser recolhidos e destinados pelo profissional responsável pelo atendimento que gerou o RSS. Os produtos relativos à revelação de lâminas de radiolo- gia podem ser submetidos ao processo de neutralização para alcançaram pH neutro (entre 7 e 9) para que possam ser descartadas em rede de esgoto, desde que atendam às diretrizes locais vigentes. Pilhas, baterias e acumuladores de energia contendo materiais pesados devem ser descartados de acordo com a Resolução do CONAMA ${ }^{\circ}$ $257 / 1999$.

Grupo C: trata sobre os rejeitos contaminados por isótopos radioativos, devendo ser segregados de acordo com a natureza física do material e do radionuclídeo presente e o tempo necessário para atingir o limite de eliminação.

Grupo D: são materiais gerais que podem ser destinados à reciclagem ou reutilização, sendo segregados em lixeiras identificadas com as respectivas cores: azul para papéis, amarelo para metais, verde para vidros, vermelho para plásticos, marrom para resíduos orgânicos. Os demais tipos de resíduos do grupo $\mathrm{D}$ podem ser coletados em recipientes de cor cinza. Caso não haja coleta seletiva no local, não é exigida a segregação dos resíduos no local.

Grupo E: são os materiais perfurocortantes, que devem ser descartados separadamente, no local de sua geração imediatamente após o uso ou necessidade de descarte, em recipientes rígidos e resistentes. Agulhas devem ser descartadas juntamente com as seringas, sendo proibido reencapá-las ou retirar manualmente. O descarte dos recipientes deve ser realizado sempre que atingir 2/3 da capacidade máxima, os resíduos gerados pelo atendimento domiciliar devem ser descartados corretamente pelos profissionais que os geraram. 
contra-se ilustrado na Figura 1, com as respecti-

Figura 1 - Classificação dos RSS

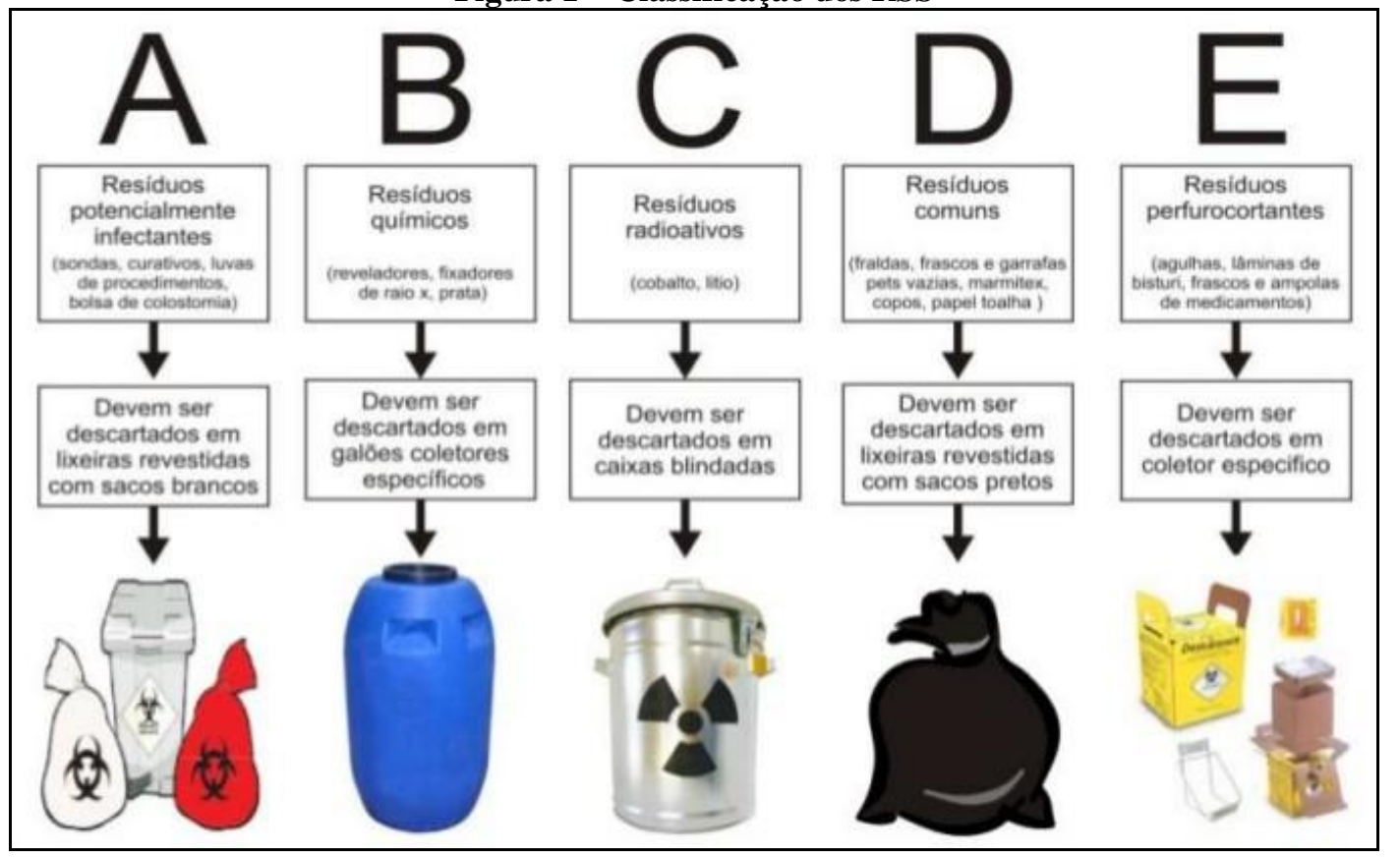

Fonte: Wons (2017).

\subsubsection{Armazenamento externo}

A RDC 306 de 2004 aponta alguns cuidados sobre o armazenamento de resíduos, quando feita na parte externa ao órgão gerador de RSS. Dentro os principais aspectos a proibição da circulação de carrinhos ou tonéis em via pública, obrigando que haja acesso interno pelas edificações do gerador do RSS.

O abrigo externo para os RSS deve ser dimensionado de acordo com o volume de resíduos gerado e de acordo com a periodicidade da coleta. O piso deve ser feito com material liso, impermeável, lavável e de fácil higienização, o fechamento deve ser feito com material de alvenaria e com aberturas para ventilação, com no mínimo a proporcionalidade de área de 1/20 em re- lação ao piso. A abertura para ventilação deve possuir grades para que evite a proliferação de insetos e animais que possam acessar os resíduos ali armazenados. Deverão haver também canaletas e ralo sifonado para facilitar a limpeza do recinto, bem como iluminação artificial.

Caso o órgão gerado de RSS não possua fluxo semanal superior a 700 litros ou diário de 150L, poderá optar pela instalação de abrigo reduzido. A diferença na construção é tão somente quanto à ventilação, que poderá ser feita por duas passagens de ar com $10 \times 20 \mathrm{~cm}$, uma com altura mínima de $20 \mathrm{~cm}$ do solo e outra com altura máxima $20 \mathrm{~cm}$ inferior ao teto do recinto.

\subsubsection{Segurança ocupacional}


Quanto à segurança ocupacional a RDC 306 de 2004 apresenta algumas preocupações quanto aos colaboradores que realizarão as atividades de descarte, segregação e destinação dos RSS. Destacam-se algumas prescrições que a RDC apresenta, os trabalhadores devem ser imunizados e conformidade com o Programa Nacional de Imunização (PNI). Os funcionários encarregados destas atividades devem ser capacitados no ato da admissão e deverão ser mantidos sob o processo de educação continuada, incluindo responsabilidade para com higiene pessoal, utilização de EPI, outrossim, deverão estar cientes do PGRSS da empresa que o contratou.

Por sua vez a educação continuada deverá ser aplicada a todos os colaboradores, independentemente do vínculo empregatício, abordando temas como: noções sobre o ciclo de vida dos produtos, conhecimento sobre a legislação ambiental e descartes de RSS; definições, tipos e classificações de RSS; conhecimento e responsabilidade das tarefas; orientações sobre a utilização de EPI; Biossegurança, higiene pessoal e do ambiente.

2.3 Resolução da diretoria colegiada (RDC) 222 de 2018

A Resolução de Diretoria Colegiada de No 222 foi aprovada em 28 de março de 2018 e publicada no Diário Oficial da União no dia posterior. Destaca-se que dispõe sobre os requisitos de Boas Práticas de Gerenciamento dos Resíduos de Serviços de Saúde. Observa-se que a RDC 222 revoga a RDC 306 a partir da entrada em vigor da nova resolução, que possui um prazo de 180 dias para tal, sendo estimado que dia 29 de junho de 2018 seja a data prevista. São apresentadas as principais mudanças em relação à RDC 306 de 2004.

Quanto à abrangência, a destinação da nova RDC engloba o rol de atividades que geram RSS descritos na RDC 306 e inclui uma nova categoria: a de salões de beleza e estética, dentre outros afins que possam gerar RSS. Outrossim, a RDC não diferencia os serviços geradores de RSS, quanto à esfera administrativa ou quanto a natureza da organização, devendo ser igualmente aplicada a todos os serviços que geram RSS.

Um aspecto que deve ser mostrado em relação à RDC anterior é devido à PNRS de 2010. A nova RDC engloba questões de logística reversa e trata de mesma forma, como um instrumento de desenvolvimento econômico e social, descrito acima, na PNRS de 2010.

Da mesma forma que a RDC anterior, a nova dispõe sobre o Plano de Gerenciamento de Resíduos de Serviços da Saúde. Desta vez abrange que além de toda organização, a geradora de RSS deva possuir o PGRSS, deve apresentar e manter arquivados documentos comprobatórios de capacitação e treinamento dos funcionários, cópia do contrato de prestação de serviços e da licença ambiental das empresas prestadoras de serviços para destinação de RSS e comprovação de operação de venda ou de doação dos RSS destinados à recuperação, à reciclagem, à compostagem e à logística reversa. É mantida a responsabilidade do serviço gerador de RSS em elaborar a PGRSS, mas é por meio da RDC 222 possibilitado que a elaboração, implantação e o monitoramento do PGRSS sejam terceirizados. 
2.3.1 Segregação, acondicionamento e identificação dos RSS

Em relação ao Grupo A, descrito anteriormente pela RDC 306 e inalterado pela RDC 222, destaca-se a flexibilização quanto ao período que pode ser utilizado os sacos para acondicionamento. Mantém o nível de segurança, que é no máximo $2 / 3$ da capacidade da embalagem, mas que poderá ser trocada em até 48h independentemente do volume. Há também a flexibilização para com as embalagens do grupo A, onde as embalagens vermelhas poderão ser substituídas pelas brancas (de mesmo material), desde que as regulamentações locais permitam.

Quanto à coleta e transporte interno, os RSS deve ser transportado atendendo a rota e horários previamente definidos. Já para o armazenamento externo, os RSS deve ser acondicionado dentro de coletores com tampa fechada.

Há uma nítida flexibilização quanto ao armazenamento externo do Grupo A, quanto à construção do abrigo local adequado, por outro lado há uma observação quanto ao armazenamento de RSS de fácil putrefação. Segundo o artigo 32 da RDC 222, caso os resíduos de fácil putrefação demorem mais de 24 para sua destinação, devem ser acondicionados a métodos de conservação, mas não é determinada como seria de fato a conservação.

Ainda é destacado que o abrigo deverá possuir área coberta para a pesagem dos RSS, quando necessário. Outro aspecto é quanto à segurança, o local deverá possuir sistema elétrico e de combate a incêndio, que atendam os requisitos de proteção estabelecidos pelos órgãos competentes. Outrossim, quanto ao transporte, os veículos para transporte externo não podem possuir sistema de compactação para que não sejam danificadas as embalagens contendo RSS, com exceção para os do grupo D.

Quanto à destinação, a nova RDC aparenta estar em consonância para com a PNRS de 2010, o primeiro (número 40) artigo já indica isso, ao definir que os RSS que não apresentem riscos biológicos, químico ou radiológicos podem ser encaminhados para reciclagem, recuperação, reutilização, compostagem, aproveitamento genético ou logística reversa. Há também a flexibilização quanto às embalagens primárias dos medicamentos, que podem ser destinadas para a logística reversa sem necessariamente passar por tratamento prévio.

Outro ponto que a antiga RDC não abarcava e agora está definido é quanto às apreensões de medicamentos ou objetos de ações de fiscalização. A correta destinação destes é de total responsabilidade do órgão que coletou tais resíduos.

Quanto às embalagens do grupo $\mathrm{B}$, há também a flexibilização, para que as embalagens de produtos químicos sem periculosidade agora poderão ser encaminhadas para processos de reciclagem. Já o descarte de pilhas, baterias, acumuladores de carga e lâmpadas fluorescentes deverão ser feitos conforme as normas vigentes, mais uma vez demonstra que a PNRS 2010 foi levada em consideração na formulação da nova RDC.

Quanto ao grupo E, que abrange os materiais perfurocortantes e escarificantes, há 
novas regulamentações que auxiliam e possibilitam reutilização de embalagens, desde que seja realizado por processo automatizado e que os recipientes sejam descontaminados para que sejam então reutilizados. Quanto às seringas, permite-se que seja utilizado processo de separação do conjunto seringa agulha, desde que seja realizado com auxílio de dispositivos de segurança, ainda é vedada a desconexão e o reencape manual de agulhas.

\subsection{Sustentabilidade e desenvolvimento sustentável}

Nas últimas décadas os efeitos sociais e ambientais que impactam a sociedade estão sendo discutidos por especialistas, acadêmicos e políticos (CLARO; CLARO; AMÂNCIO, 2008; LADERA; VERA; TRIGUEIROS, 2014). Nesse contexto, os termos sustentabilidade e desenvolvimento sustentável se encontram cada vez mais presentes nessas discussões, recebendo a atenção mundial. Como observado por Axelsson et al. (2011), a sustentabilidade e o desenvolvimento sustentável ganharam aceitação em nível global devido aos desafios e preocupações enfrentados em temas como o desenvolvimento rural, a conservação ambiental, mudança climática, questões sobre a energia e o bem-estar da população, entre outras questões.

A sustentabilidade pode ser entendida como uma característica de um processo ou sistema que pode manter-se por tempo indefinido (PEREIRA; SILVA; CARBONARI, 2011). Já o conceito de desenvolvimento sustentável mais difundido foi o definido no relatório nomeado Our Common Future (Nosso Futuro Comum), conhecido também como relatório da Comissão Brundtland de 1987, o qual considera que o desenvolvimento sustentável é o desenvolvimento que satisfaz as necessidades do presente sem comprometer a capacidade de atender às necessidades das gerações futuras (WCED, 1987). Essa definição enfatiza um dos pressupostos básicos de sustentabilidade, a visão de longo prazo, uma vez que inclui os interesses das futuras gerações. De maneira complementar Sartori et al. (2014) afirma que a sustentabilidade pode ser considerada um mecanismo para alcançar o desenvolvimento sustentável aspirado. Além disso, para Axelsson et al. (2011), o desenvolvimento sustentável se trata de um processo coletivo da sociedade que envolve múltiplos stakeholders. Incorporado ao desenvolvimento sustentável, se destaca o conceito do Triple Bottom Line, que consiste na sustentabilidade econômica, ambiental e social (OLAWUMI; CHAN, 2018; LADERA; VERA; TRIGUEIROS, 2014).

O conceito do Triple Bottom Line foi apresentado por Elkington no livro Cannibals with Forks: The Triple Bottom Line of 21stCentury Business, em 1997 (ELKINGTON, 2012). Esse conceito parte de uma metáfora onde cada um dos três dentes de um garfo representam as dimensões, econômica, ambiental, e social, procurando assim, responder ao seguinte questionamento: $\mathrm{O}$ capitalismo, da mesma forma que um canibal, se tornaria civilizado se utilizasse garfo? (ELKINGTON, 2012). Na visão do autor a operacionalização da sustentabilidade requer a integração das três dimensões, que devem ser 
harmonizadas para alcançar os objetivos desejados.

Para Júnior et al. (2011) estas três dimensões são interdependentes, se relacionam mutuamente e devem estar integradas para a tomada de decisões sobre o desenvolvimento futuro. Nesse sentido, a dimensão econômica considera o uso eficiente de recursos sem comprometer o crescimento econômico ou a lucratividade (ELKINGTON 2012). Essa dimensão não inclui só a economia formal, mas também as atividades informais que contribuem para a elevação da renda monetária e o padrão de vida dos indivíduos (CLARO; CLARO; AMÂNCIO, 2008). A dimensão ambiental inclui a utilização dos recursos de forma racional e permanente, com o objetivo de reduzir os impactos, industriais (ELKINGTON, 2012), desse modo, a sustentabilidade ambiental está preocupada com a conservação ambiental e com a limitação da atividade humana, que deve considerar a capacidade de suporte do ecossistema (OLAWUMI; CHAN, 2018). Já a dimensão social inclui a questão da justiça social, para que seja desenvolvido um mundo mais justo por meio das relações com todos os stakeholders, além da elevação da qualidade de vida e da equidade social (ELKINGTON, 2012). A dimensão social se concentra no bem-estar social das pessoas, defende o equilíbrio entre as necessidades de um indivíduo com as necessidades do grupo (OLAWUMI; CHAN, 2018).

Schalch, Castro e Córdoba (2017) ressaltam que a sustentabilidade por si só, não é uma meta a ser atingida, se trata de um caminho a ser percorrido. Para tanto serão necessários passos firmes que conduzam a melhorias continuas de processos, produtos e atitudes. Ainda de acordo com o autor a gestão integrada de resíduos sólidos oferece uma alternativa viável para se atingir uma condição mais sustentável, uma vez que não contribui apenas com o desempenho ambiental, mediante diminuição no volume dos resíduos e da periculosidade dos resíduos gerados, mas também no desempenho econômico, por permitir identificar desperdícios de material e processos ineficientes, entre outros aspectos.

Corroborando Besen et al. (2010) destaca que a gestão e a disposição inadequada dos resíduos sólidos causam impactos socioambientais, tais como degradação do solo, comprometimento dos corpos d'água e mananciais, intensificação de enchentes, contribuição para a poluição do ar e proliferação de vetores de importância sanitária nos centros urbanos.

Cabe destacar, que existem alguns autores que ampliam a visão de sustentabilidade incorporando outras dimensões além do Triple Bottom Line, como Sachs (2002), que descreve oito dimensões da sustentabilidade: social, cultural, ecológica, ambiental, territorial, econômica, política nacional e política internacional. No entanto, para o presente estudo adota-se o conceito tradicional de sustentabilidade, considerando as dimensões econômica, social e ambiental. 


\section{METODOLOGIA}

A metodologia é um conjunto de técnicas e procedimentos que busca viabilizar a execução da pesquisa (JUNG, 2004) e tem o objetivo de ultrapassar o senso comum através do método científico (MANAYO, 2008). Quanto ao caminho metodológico, o presente estudo se define como de natureza exploratória e quanto aos procedimentos foi adotada a análise documental. Malhotra (2011) sugere que pesquisas exploratórias são relevantes quando há necessidade de ter uma visão mais clara sobre um fato, tendo como finalidade formular um problema ou defini-lo com maior precisão, obter critérios para desenvolver uma abordagem do problema e estabelecer prioridades para pesquisas posteriores. Corroborando, Gil (2007) afirma que as pesquisas exploratórias têm o intuito de aumentar o conhecimento em relação a um determinado fenômeno, explorando uma determinada realidade.

Gil (2007) afirma que a pesquisa documental se assemelha muito à pesquisa bibliográfica. A diferença essencial entre ambas está na natureza das fontes, enquanto a pesquisa bibliográfica se utiliza fundamentalmente das contribuições dos diversos autores sobre determinado assunto, a pesquisa documental utiliza materiais que ainda não receberam um tratamento analítico, ou que ainda podem ser reelaborados de acordo com os objetos da pesquisa.

Os documentos analisados foram legislações brasileiras que versam sobre o gerenciamento de resíduos de saúde, conforme Quadro 1. A análise dos dados ocorreu de forma comparativa entre as duas legislações, assim foram consideradas as mudanças detectadas entre ambas, bem como a relação com as dimensões do desenvolvimento sustentável.

Quadro 1 - Resoluções analisadas

\begin{tabular}{|l|l|}
\hline Resolução & \multicolumn{1}{|c|}{ Tema } \\
\hline $\begin{array}{l}\text { RDC n. 306 de } \\
\text { de de } 2004\end{array}$ & $\begin{array}{l}\text { Dispõe sobre o regulamento } \\
\text { técnico para o gerenciamento de } \\
\text { resíduos de serviços de saúde. }\end{array}$ \\
\hline $\begin{array}{l}\text { RDC n. 222 de } 28 \text { de março de } \\
2018\end{array}$ & $\begin{array}{l}\text { Dispõe sobre os requisitos de } \\
\text { boas práticas de gerenciamento } \\
\text { dos resíduos de serviços de } \\
\text { saúde. }\end{array}$ \\
\hline
\end{tabular}

Fonte: Elaborado pelos autores.

\section{ANÁlISE E DISCUSSÃO DOS RESULTADOS}

O presente estudo teve o objetivo de realizar uma análise crítica da Resolução da Diretoria Colegiada (RDC) 222/2018 à luz das dimensões do desenvolvimento sustentável (ambiental, social e econômica) a fim de verificar os avanços e desafios da legislação.

Inicialmente, o gerenciamento de resíduos de serviços de saúde era regulamentado somente pelo Conselho Nacional do Meio Ambiente (CONAMA), e a partir da Lei 9.782/1999 foi criada a Agência Nacional de Vigilância Sanitária (ANVISA) que passou a regulamentar os procedimentos internos de serviços de saúde.

A RDC 222/2018 surgiu com a necessidade de atualização da resolução anterior, respondendo a questionamentos existentes, evolução das tecnologias e a entrada em vigor da Lei 12.305/2010 que instituiu a Política Nacional de Resíduos Sólidos (PNRS). A Figura 2 traz uma síntese dos avanços e desafios da nova legislação, em que se considera: desafios existentes (Vermelho), avanços de baixo impacto (Amarelo) e avanços de alto impacto (Verde). 
Figura 2 - Painel resumo das principais características da RDC-222\2018

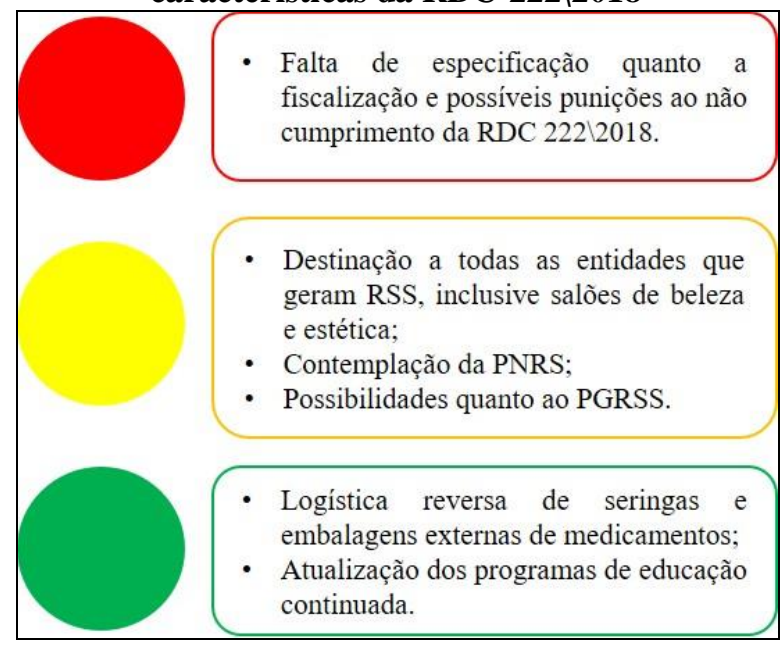

Fonte: Elaborado pelos autores.

Os quadrantes apresentados na Figura 2 são uma síntese dos avanços e desafios da RDC 222\2018. No quadrante vermelho, como principais desafios tem-se a necessidade do rigor no cumprimento, fiscalização e possíveis punições às entidades que não se ajustarem à nova RDC. Como avanços de baixo impacto, situados no quadrante amarelo, estão às novas classes de serviços da saúde vinculados: salões de beleza e estética, que não estavam inclusos na RDC 306/2004. Ainda como avanços de baixo impacto estão a contemplação da PNRS de 2010 na formulação da RDC 222, bem como, uma nova possibilidade quanto ao PGRSS, em que as organizações de serviços da saúde podem terceirizar a formulação e implementação do programa, o que pode acarretar em maior agilidade e menores custos à organização.

Quanto a avanços considerados de alto impacto está a contemplação da possibilidade de logística reversa das embalagens externas de medicamentos e seringas, desde que estas sejam separadas das agulhas de forma mecanizada, evitando assim, riscos aos colaboradores. Outro avanço interessante é a reformulação dos programas de educação continuada, contemplando o treinamento contínuo dos colaboradores, não distinguindo os colaboradores quanto ao vínculo empregatício.

Da mesma forma, se buscou identificar na nova Resolução da Diretoria Colegiada (RDC) 222/2018 o que contempla das dimensões do desenvolvimento sustentável (ambiental, social e econômica). O Quadro 2 apresenta síntese dos aspectos contemplados pela legislação referentes as dimensões do desenvolvimento sustentável.

Quadro 2 - Síntese dos aspectos das dimensões do desenvolvimento sustentável contemplados pela RDC-222\2018

\begin{tabular}{|l|c|c|c|}
\hline $\begin{array}{l}\text { Aspectos da Ambienta } \\
\text { RDC 22212018 }\end{array}$ & Social & Econômica \\
\hline $\begin{array}{l}\text { Minimização de } \\
\text { resíduos }\end{array}$ & $\mathrm{X}$ & $\mathrm{X}$ & $\mathrm{X}$ \\
\hline $\begin{array}{l}\text { Encaminhament } \\
\text { o seguro e } \\
\text { eficiente }\end{array}$ & $\mathrm{X}$ & $\mathrm{X}$ & $\mathrm{X}$ \\
\hline $\begin{array}{l}\text { Proteção dos } \\
\text { trabalhadores }\end{array}$ & & $\mathrm{X}$ & $\mathrm{X}$ \\
\hline $\begin{array}{l}\text { Preservação da } \\
\text { saúde pública }\end{array}$ & $\mathrm{X}$ & & \\
\hline $\begin{array}{l}\text { Preservação dos } \\
\text { recursos naturais } \\
\text { e meio ambiente }\end{array}$ & & & \\
\hline
\end{tabular}

Fonte: Elaborado pelos autores.

Ao definir o gerenciamento dos resíduos de serviços de saúde (GRSS) como um conjunto de procedimentos de gestão com o objetivo de minimizar a geração de resíduos e proporcionar um encaminhamento seguro, de forma eficiente, visando à proteção dos trabalhadores e a preservação da saúde pública, dos recursos naturais e do meio ambiente (ANVISA, 2004; ANVISA, 2018), pode-se constatar uma preocupação com as três dimensões do desenvolvimento sustentável. 
Para contribuir com a minimização de resíduos a legislação considera o próprio Plano de Gerenciamento como uma ferramenta capaz de ajudar a diminuir a quantidade de resíduos a serem tratados, uma vez que com o planejamento, a adequação dos procedimentos de manejo, o sistema de sinalização e o uso de equipamentos apropriados, os gestores poderiam acompanhar indicadores e realizar as melhorias necessárias.

Além disso, com um correto manejo, seria possível promover o reaproveitamento de parte dos resíduos, mediante segregação dos materiais recicláveis, reduzindo os custos de tratamentos desnecessários e ainda os custos relacionados à disposição final. Cesaro e Belgiorno (2017) destacam que resíduos perigosos, como o infeccioso, são geralmente gerenciados a custos mais elevados do que os resíduos não perigosos. De acordo com Makajic-Nikolic et al. (2016) a segregação inadequada dos resíduos na fonte é determinante na contaminação de resíduos não perigosos pelos resíduos perigosos, aumentando assim a ocorrência de lesões no trabalho.

Outro ponto importante para a diminuição dos resíduos é a educação e conscientização das pessoas envolvidas no manejo dos resíduos. Considerando esse aspecto, a legislação aponta que as formas de reduzir a geração de RSS e a reutilização de materiais deve ser um dos tópicos tratados no programa obrigatório de educação continuada. Portanto, todos os trabalhadores envolvidos nas atividades de gerenciamento de resíduos devem estar informados sobre tal aspecto.

Nesse sentido, Mosquera et al. (2014) apontou que, após uma intervenção educativa em um hospital espanhol, a produção de resíduos infecciosos foi reduzida pela metade e, apesar de uma redução geral na produção de resíduos, constatou-se uma quantidade maior de resíduos não perigosos. Em outro estudo, Moreira e Günther (2013) monitoraram a variação decorrente da implementação do plano de gerenciamento de resíduos de serviços de saúde em um centro brasileiro de atenção primária à saúde, baseando-se na aplicação de uma lista de verificação dos requisitos legalmente estabelecidos para a gestão dos resíduos. Os resultados encontrados em um primeiro momento constataram o aumento total na geração de resíduos, entretanto, a distribuição percentual em categorias mudou para uma composição mais favorável sob o ponto de vista ambiental e sanitário. Foi observada uma redução dos resíduos não recicláveis e um aumento na segregação de material reciclável, além da reutilização de resíduos orgânicos para compostagem, e uma redução na taxa de geração de resíduos infecciosos em áreas críticas.

Deste modo, a minimização de recursos abarca questões tanto ambientais, como sociais e econômicas. Na dimensão ambiental, o correto manejo dos resíduos contribui com a diminuição de riscos de contaminação ao meio ambiente, diminui também a necessidade do uso de materiais não renováveis na produção de novos produtos, quando estes podem ser fabricados com a utilização de materiais recicláveis. Por outro lado, na dimensão social a principal relação se dá com a proteção da saúde dos trabalhadores e da população, e na dimensão econômica ao reduzir os custos provenientes de uma gestão inadequada. 
De forma similar o encaminhamento seguro e eficiente proporciona benefícios às três dimensões destacadas. Ao promover as boas práticas do gerenciamento dos resíduos, a saúde dos trabalhadores é um dos fatores beneficiados, que por sua vez está diretamente relacionado com a dimensão social, reduzindo os riscos de doenças que poderiam ser ocasionadas, ajudando assim, a contribuir com o bem-estar dos trabalhadores.

Graikos et al. (2010) confirmam que a gestão inadequada envolve um alto risco de contaminação ambiental por tratamento e disposição inadequados, assim como o risco de infecção e ferimentos ao pessoal em contato com este resíduo devido à possível transmissão de infecções transmitidas pelo sangue. Outro estudo realizado por Akpieyi,Tudor e Dutra (2015) estimou que as exposições que ocorrem com maior frequência envolvem contato com materiais perfurocortantes, agentes infecciosos e substâncias perigosas e ferimentos pessoais durante o manuseio de resíduos, principalmente nas enfermarias (em torno de $65 \%$ do total de exposições). No entanto, a possível contaminação dos resíduos de forma geral com agentes infecciosos durante o manejo inadequado apresenta riscos ainda mais amplos para o meio ambiente e a saúde pública.

Da mesma forma, com um adequado gerenciamento dos resíduos a saúde da população como um todo também é preservada, impactando na dimensão social e também na econômica, considerando os altos gastos que podem ser economizados com o sistema de saúde pública, em casos de tratamento por exposições a resíduos perigosos. $\mathrm{O}$ encaminhamento seguro e eficiente também proporciona a preservação dos recursos naturais e do meio ambiente. A RDC 222/2018 prevê que os diferentes RSS que não apresentam risco biológico, químico ou radiológico possam ser encaminhados para reciclagem, recuperação, reutilização, compostagem, aproveitamento energético ou logística reversa (ANVISA, 2018), o que resulta em impactos positivos diretos na dimensão ambiental.

Ao mesmo tempo para realizar todas as atividades de gerenciamento dos resíduos são necessárias diversas atividades econômicas, envolvendo diferentes empresas e trabalhadores. Contribuindo para a geração de renda e o desenvolvimento local.

Sendo assim, a partir da análise reflexiva dos desafios, avanços de baixo impacto, avanços de alto impacto da Resolução da Diretoria Colegiada (RDC) 222/2018 e os aspectos contemplados nas dimensões do desenvolvimento sustentável, observou-se inicialmente uma mudança de postura em relação ao objetivo da nova resolução, que ampliou as discussões de "regulamento técnico para o gerenciamento de resíduos de serviços de saúde" para "requisitos de boas práticas de gerenciamento dos resíduos de serviços de saúde".

A nova legislação proporcionou uma atualização necessária, principalmente para a inclusão de práticas preconizadas pela Política Nacional de Resíduos Sólidos (PNRS), que foi criada seis anos após a Resolução da Diretoria Colegiada (RDC) 306/2004, e ainda, é necessária uma reflexão quanto à fiscalização e cumprimento da legislação, que é uma lacuna existente. Evidenciou-se também um equilíbrio entre os 
aspectos das dimensões do desenvolvimento sustentável (ambiental, social e econômica) contemplados pela nova resolução, com predomínio da dimensão social.

\section{CONSIDERAÇÕES FINAIS}

Buscou-se com o presente estudo realizar uma análise crítica da Resolução da Diretoria Colegiada (RDC) 222/2018 à luz das dimensões do desenvolvimento sustentável (ambiental, social e econômica) a fím de verificar os avanços e desafios da legislação. Este objetivo foi plenamente atingido, conforme apresentado em síntese na Figura 2 e Quadro 2.

Ficou evidente que os principais avanços da nova legislação foram a atualização necessária em detrimento a Política Nacional de Resíduos Sólidos (PNRS) criada no ano de 2010 e a mudança do objetivo da legislação para "requisitos de boas práticas de gerenciamento dos resíduos de serviços de saúde", e como principal lacuna visualizou-se a fiscalização e cumprimento da legislação. Sobre as dimensões do desenvolvimento sustentável (ambiental, social e econômica) contemplados pela nova resolução observou-se equilíbrio e predomínio do aspecto social.

Conclui-se que a nova RDC-222/2018 é um avanço importante em busca da regulamentação e das boas práticas no tratamento de resíduos de serviços de saúde em convergência as dimensões do desenvolvimento sustentável, apesar da dificuldade na fiscalização e cumprimento da legislação.

O estudo apresenta como limitação a restrição quanto à pesquisa documental nas legislações vigentes, e como sugestão para estudos futuros tem-se a inclusão de coleta de dados com gestores da área da saúde e órgãos fiscalizadores, e pesquisa de legislação e boas práticas em países desenvolvidos.

\section{REFERÊNCIAS}

ABRELPE, Panorama 2016. Disponível em: <http://www.abrelpe.org.br/Panorama/panorama2016 .pdf> Acesso em: 25 de jun. de 2018.

AKPIEYI, A.; TUDOR, T. L.; DUTRA, C. The utilisation of risk-based frameworks for managing healthcare waste: A case study of the National Health Service in London. Safety Science, v. 72, p. 127132, 2015.

ANVISA. Agência Nacional de Vigilância Sanitária. Resolução RDC n. 306 de 7 de dezembro de 2004: Dispõe sobre o regulamento técnico para o gerenciamento de resíduos de serviços de saúde. Diário Oficial da União, 2004.

ANVISA. Agência Nacional de Vigilância Sanitária. Resolução RDC n. 222 de 28 de março de 2018: Dispõe sobre os requisitos de Boas Práticas de Gerenciamento dos Resíduos de Serviços de Saúde. Diário Oficial da União, 2018.

AXELSSON, R. et al. Sustainable Development and Sustainability: Landscape Approach as a Practical Interpretation of Principles and Implementation Concepts, Journal of Landscape Ecology, v. 4, n.3, p. 5-30, 2011.

BRASIL. Lei n. 12.305, de 2 de agosto de 2010. Institui a Política Nacional de Resíduos Sólidos altera a Lei no 9.605, de 12 de fevereiro de 1998, e dá outras providências. Diário Oficial da União, 2010.

BESEN, G. R. et al. Resíduos sólidos: vulnerabilidades e perspectivas. In: SALDIVA P. et al. Meio ambiente e saúde: o desafio das metrópoles. São Paulo: Ex Libris, 2010.

CLARO, P. B. DE O.; CLARO, D. P.; AMÂNCIO, R. Entendendo o conceito de sustentabilidade nas organizações. Revista de Administração, v. 43, n. 4, p. 289-300, 2008.

CESARO, A.; BELGIORNO, V. Sustainability of Medical Waste Management in Different Sized Health Care Facilities. Waste and Biomass Valorization, v. 8, n. 5, p. 1819-1827, 2017.

DAPPER, Paloma Veridiane; GRENZEL, Rafael; ZANATTA, Jocias Maier. Estrutura organizacional da manutenção, escolha do método de trabalho e os indicadores de gestão de pessoas: uma abordagem 
conceitual. Revista da Universidade Vale do Rio Verde, v. 16, n. 1, 2018.

ELKINGTON, J. Canibais com garfo e faca. São Paulo: M. Books do Brasil Ltda, 2012.

GRAIKOS, A. et al. Composition and production rate of medical waste from a small producer in Greece. Waste Management, v. 30, n. 8-9, p. 1683-1689, 2010 .

GIL, A. C. Métodos e técnicas de pesquisa social. São Paulo: Atlas, 2007.

JÚNIOR, A. C. S, et al. Políticas públicas, tecnologias limpas e sustentabilidade: MDL em parques eólicos no Brasil. REUNA, Belo Horizonte, v.16, n.2, mai./jun. 2011.

SCHALCH, V.; CASTRO, M. A. S.; CÓRDOBA, R. E. Gestão Sustentável de Resíduos Sólidos. In: JR., P. A., SAMPAIO, C. C, FERNANDES, V. (eds.). Gestão Empresarial e sustentabilidade, Barueri: Manole, 2017.

KARLINER, J.; GUENTHER, R. Agenda Global Hospitais Verdes e Saudáveis. Uma agenda abrangente de saúde ambiental para hospitais e sistemas de saúde em todo o mundo, 2013. Disponível em: <http://greenhospitals.net/wpcontent/uploads/2012/03/GGHHA-Portugese.pdf $>$ Acesso em: 27 de jun. de 2018.

JUNG, Carlos Fernando. Metodologia para pesquisa e desenvolvimento - aplicada a novas tecnologias, produtos e processos. Rio de Janeiro: Axcel books do Brasil Editora Ltda, 2004.

LADERA, R.; VERA, L. A. R.; TRIGUEIROS, R. E. Gestão dos resíduos sólidos e logística reversa : um estudo de caso em uma organização do setor de construção civil. Gestão \& Planejamento, v. 15, n. maio/ago., p. 283-304, 2014.

MAKAJIC-NIKOLIC, D. et al. The fault tree analysis of infectious medical waste management. Journal of Cleaner Production, v. 113, p. 365-373, 2016.

MALHOTRA, N. K. Pesquisa de Marketing: foco na decisão. 3. ed. São Paulo: Pearson Prentice Hall, 2011.

MINAYO, Cecília S. (organizadora). Pesquisa Social: Teoria, método e criatividade. 27. ed. Petrópolis: Vozes, 2008.

MOREIRA, A. M. M.; GÜNTHER, W. M. R. Assessment of medical waste management at a primary health-care center in São Paulo, Brazil. Waste Management, v. 33, n. 1, p. 162-167, 2013.

MOSQUERA, M. et al. Evaluation of an education and training intervention to reduce health care waste in a tertiary hospital in Spain. American Journal of Infection Control, v. 42, n. 8, p. 894-897, 2014.

NOGUEIRA, D. N. G.; CASTILHO, V. Resíduos de serviços de saúde: mapeamento de processo e gestão de custos como estratégias para sustentabilidade em um centro cirúrgico. REGE-Revista de Gestão, v. 23, n. 4, p. 362-374, 2016.

OLAWUMI, T. O.; CHAN, D. W. M. A scientometric review of global research on sustainability and sustainable development. Journal of Cleaner Production, v. 183, p. 231-250, 2018.

PEREIRA. A. C; SILVA, G. Z. da; CARBONARI, M. E. E. Sustentabilidade, responsabilidade social e meio ambiente. $1^{\mathrm{a}}$ Ed. São Paulo: Saraiva, 2011.

SACHS, I. Caminhos para o desenvolvimento sustentável. 3. ed. Rio de Janeiro: Garamond, 2002.

SARTORI, S. et al. Sustainability and sustainable development: A taxonomy in the field of literature. Ambiente \& Sociedade, v. XVII, n. 1, p. 1-20, 2014.

\section{WCED, WORLD COMISSION ON}

ENVIROMENTAL AND DEVELOPMENT. Our common future. Oxford: Oxford University Press, 1987.

WONS, F. Plano de Gerenciamento de Resíduos de Serviços de Saúde - PGRSS. Disponível em: $<$ http://licenciadorambiental.com.br/plano-degerenciamento-de-residuos-de-servicos-de-saudepgrss/> Acesso em: 27 de jun. de 2018

\section{Diéssica de Oliveira Dias}

Mestranda em Administração e Graduada em Administração pela Universidade Federal de Santa Maria - UFSM.

\section{Ismael Alan Halberstadt}

Mestrando em Administração e Graduado em Administração pela Universidade Federal de Santa Maria - UFSM.

\section{Jocias Maier Zanatta \\ Doutorando em Administração pela Universidade Federal de Santa Maria - UFSM, Mestre em Desenvolvimento pela Universidade Regional do Noroeste do Estado do Rio Grande do Sul - UNIJUI, Especialização em Gestão Financeira, Controladoria e Auditoria e Graduação em Administração pela Sociedade Educacional Três de Maio - SETREM. Professor e Administrador hospitalar.}

Marcelo Trevisan
Doutor em Administração pela Universidade Federal
do Rio Grande do Sul - UFRGS, Mestre em


Administração pela Universidade Federal de Santa Catarina - UFSC, Especialista em Administração Gerência de Serviços e Graduado em administração pela Universidade Federal de Santa Maria - UFSM. Professor da Universidade Federal de Santa Maria UFSM. 